\title{
Erratum to: Modelling LARES temperature distribution and thermal drag
}

Phuc H. Nguyen ${ }^{\mathrm{a}}$ and Richard Matzner ${ }^{\mathrm{b}}$

Department of Physics and Texas Cosmology Center, The University of Texas, Austin, TX 78712, USA

Original article: Eur. Phys. J. Plus (2015) 130: 206, DOI: 10.1140/epjp/i2015-15206-2

Received: 4 April 2016

Published online: 31 May 2016 - (C) Società Italiana di Fisica / Springer-Verlag 2016

1) In footnote 1 , page 2 , the notations of parameters in eq. (2) are wrong, i.e.

$-I$ is the moment of inertia of the gyroscope,

should read

$I$ is the moment of inertia of Earth,

- $\boldsymbol{S}$ is its angular momentum.

should read

$\boldsymbol{S}$ is Earth's angular velocity.

2) In eq. (49), sect. 7.3, the second symbol $\alpha_{\mathrm{gl} \text {,vis }}$ (the absorptivity of glass in the visible) should read $\alpha_{W, \text { vis }}$ (the absorptivity of the metal in the visible). The equation is then corrected as

$$
P_{W, \mathrm{vis}}=\alpha_{W, \mathrm{vis}} \pi R_{\mathrm{sat}}^{2} \Phi-\alpha_{W, \mathrm{vis}} \Phi \pi R^{2}+\left[\frac{1}{2}\left(1-\alpha_{\mathrm{gl}, \mathrm{vis}}\right)-\alpha_{W, \mathrm{vis}}\right] \pi R^{2} \Phi \sum_{I \neq 1} n_{I} \cos \theta_{I}
$$

This is only a typo in the equation and does not change the conclusion of the paper, because in our code we used the correct quantity.

\footnotetext{
a e-mail: phn229@physics.utexas.edu

b e-mail: matzner2@physics.utexas.edu
} 